\title{
«Электронное государство» как междисциплинарная научная область: наукометрический анализ
}

\author{
А.В. Чугунов ${ }^{1}$, Ю.А. Кабанов ${ }^{1,2}$ \\ ${ }^{1}$ Университет ИТМО, \\ ${ }^{2}$ Национальный исследовательский университет «Высшая школа экономики» \\ chugunov@itmo.ru, ykabanov@hse.ru
}

\section{Аннотация}

Объектом представляемого в настоящей статье исследования является междисциплинарная научная область, условно обозначенная как «Электронное государство». Решаются две задачи - оценка концептуальной целостности данного предметного поля и изучение взаимосвязи между основными исследовательскими контекстами, особенно в связи с появлением новых междисциплинарных научных направлений, таких как электронное участие. Для решения этих задач применен наукометрический подход и автоматизированный анализ публикаций как на английском (база данных Web of Science), так и на русском языках (РИНЦ eLibrary.ru). Для кластеризации и визуализации данных использовалось программное обеспечение VOSviewer, позволяющее обеспечить визуализацию больших наборов библиометрических данных методом построения библиометрических карт.

Полученные данные свидетельствуют о том, что глобальная повестка представляет собой согласованную область, хотя важна дальнейшая интеграция между исследовательскими контекстами. В то же время, российские исследования отстают из-за низкого уровня взаимодействия с международными научными школами и отсутствия внутренних стимулов и исследовательских проектов для генерации знаний.

Ключевые слова: электронное государство, науковедение, библиометрия, научные публикации, Web of Science, РИНЦ

Библиографическая ссылка: Чугунов А.В., Кабанов Ю.А. «Электронное государство» как междисциплинарная научная область: наукометрический анализ // Государство и граждане в электронной среде. Выпуск 3 (Труды XXII Международной объединенной научной конференции «Интернет и современное общество», IMS-2019, Санкт-Петербург, 19 - 22 июня 2019 г. Сборник научных трудов). - СПб: Университет ИТМО, 2019. С. 11 - 24. DOI: 10.17586/2541-979X-2019-3-11-24

\section{1. Введение}

В поле зрения данной статьи - междисциплинарная научная область исследований «электронного правительства» и смежных концептов («электронная демократия», «электронное участие», «открытое правительство» и пр.), иногда обобщаемых термином «электронное государство». Позиционирование этого научного поля является предметом широкого и постоянного обсуждения, что вызывает интерес к оценке его концептуальной целостности и поиску взаимосвязей между основными исследовательскими контекстами. Особенно это актуально в связи с появлением новых междисциплинарных научных направлений, таких как «электронное участие» (e-participation), а также в связи с постепенным формированием исследовательских коллективов по данной тематике в России. 
Данная работа посвящена, во-первых, рассмотрению предметного поля и паттернов институционализации «электронного государства» в международной научной повестке, вовторых, ее сравнению с национальной исследовательской повесткой России. Для этого мы используем наукометрический анализ по ключевым словам публикаций (co-occurrence analysis) и их аффилиации (bibliographic coupling), что позволяет сопоставить тематические и дисциплинарные «очаги» исследований, а также их институциональную принадлежность.

\section{2. «Электронное государство» как междисциплинарная научная область}

Несмотря на внутреннюю дифференциацию тематики «электронного государства», данное научное направление имеет общие черты, связанные с его происхождением и развитием. Первой чертой является прикладной характер исследований, ориентированный на сопровождение проектов по внедрению ИКТ в деятельность органов власти, с целью повышения эффективности их функционирования и оптимизации административных процессов. Первые проекты в сфере электронного правительства стартовали в США еще в 1990-х годах, когда были приняты документы и планы стратегического характера. Следует отметить, что само явление тогда именовалось Digital Government. Фактически в это же время возникла потребность в создании первых исследовательских центров, которые также были созданы в американских университетах (Университет Штата Нью-Йорк в Олбани, Университет Вашингтона и др.) [1]. Одновременно исследователи из области политических наук и коммуникативистики обратились к проблемам трансформации демократии под влиянием ИКТ, что вызывало к жизни терминологию в стиле digital (electronic) democracy.

В европейском научном дискурсе большую популярность приобрел термин Electronic Government (E-Government, eGov), поскольку в Евросоюзе во второй половине 1990-х стала формироваться программа eEurope, где построение электронного правительства рассматривалось как один из компонентов, и где был предусмотрен регулярный мониторинг усилий государств по его развитию, продолжающийся и сейчас как eGovernment Benchmark [2]. Естественно, что европейские университеты и исследовательские центры активно включились в реализацию проектов в данной сфере. Существенное значение имели программы, стимулирующие сетевую коллаборацию между институтами ЕС и других стран, в том числе и России. Можно упомянуть такие проекты, как DemoNet, eGovMoNet, eGovPoliNet и др. [3].

В Российской Федерации первые исследования проблематики электронного правительства в 1990-х годах осуществлялись в рамках темы «Информационное общество» [4], в существенной степени стимулируемой опытом европейских программ, а также активизацией интереса к данной теме после подписания Россией «Окинавской хартии», где был обозначен курс на формирование национальных программ, что привело к принятию Федеральной целевой программы «Электронная Россия». Институционализация темы «Электронное правительство» и явная заинтересованность ИКТ-компаний в разработке информационных систем для госсектора, существенно стимулировали российское научное и экспертное сообщество к участию в проработке как концептуальных, так и прикладных вопросов, связанных с проектированием и внедрением решений «электронного правительства». Следует отметить, что многие направления исследований на стыке информатики и социальных проблематики формировались еще в Советском Союзе и этот потенциал в первоначальный период также использовался научным и экспертным сообществом [5] [6].

Постоянное географическое и тематическое расширение проблематики приводит к росту актуальности систематических обзоров литературы, которые бы оценивали зрелость поля в целом [7], или его отдельных дисциплин [8]. Многие исследователи подчеркивают фрагментацию научной области, что вполне естественно в силу разного профессионального бэкграунда ученых [9]. По мнению Х. Шолля, такая дифференциация может даже быть на 
пользу, если из разрозненной области работ по схожей тематике исследования электронного правительства превратятся в «интегративную науку» [10], В этой связи представляется важным выяснить, в какой степени такие «интегративные» тенденции действительно присутствуют, и выявить точки пересечения разных исследовательских контекстов.

В то же время, следует обратить внимание и на национальное измерение производства научного знания, противопоставив страновую динамику глобальным тенденциям. Работ, посвященных отдельным государствам или регионам, становится все больше $[11,12]$. Так, в недавней статье Р.В. Ерженина [13] подчеркивается ряд особенностей изучения электронного правительства в России, в частности, его концентрация на экономике, политологии и праве, в отличие от международной науки.

Одним из многообещающих вариантов изучения предметного поля является наукометрический анализ, приобретающий все большую популярность в исследованиях «электронного государства». Авторы, используя различные выборки данных и программное обеспечение, в основном концентрируются на анализе временной динамики для выявления наиболее важные направления исследований $[14,15,16]$, а также на выявлении моделей коллаборации между разными институтами и странами $[17,18]$. Вместе с тем, в данной области по-прежнему существуют отдельные «лакуны». Главным образом они связаны с демаркацией научной области, что проявляется в разных подходах к отбору публикаций по ключевым словам и типам. Мы полагаем, что важно охватить широкую сферу «электронного государства», используя такие ключевые слова как «электронное правительство», «электронное управление», «электронное участие» и «электронная демократия», поскольку они могут использоваться авторами как маркер, позволяющий отнести их работы к данной области, позволяющий сохранять своеобразную «идентичность» дисциплины. Кроме того, мы включаем в выборку не только журнальные статьи, но и материалы конференций, которые, как утверждаются, имеют для данной области не меньшее значение [16, 19].

\section{3. Дизайн исследования}

Как было отмечено выше, в данной работе проводится наукометрический анализ публикаций, целью которого является, с одной стороны, рассмотрение тематической и институциональной «картины» предметных областей, связанных с развитием темы «электронное государство», с другой стороны, проведение сравнительного сопоставления мирового потока научных публикаций с российским. Информационной базой исследования является метаинформация, полученная из баз данных научных публикаций, как на английском, так и на русском языке.

Для кластеризации и визуализации данных было решено использовать программное обеспечение VOSviewer (http://www.vosviewer.com), главным преимуществом которого является возможность наглядной визуализации больших наборов библиометрических данных [20]. VOSviewer имеет развитый инструментарий для построения библиометрических карт, формируя их в зависимости от заданных позиций: ассоциативный анализ по совпадению набора ключевых слов, взаимного цитирования авторами других авторов, библиографического сочетания (по спискам литературы к публикациям) и др. Карты строятся таким образом, чтобы «расстояние между двумя предметами отражало сходство предметов как можно точнее» [21, p. 5].

Следует отметить, что в анализе использовалась база данных Web of Science, что несколько сузило круг источников, т.к. имеется несколько значимых рецензируемых международных научных конференций (ICEGOV, Dg.o, EGOSE), которые публикуются в изданиях, индексируемых в базе Scopus, но отсутствуют в Web of Science. В дальнейшем планируется расширить базу данных с целью включения дополнительных ресурсов.

Для отбора метаинформации из базы данных Web of Science был использован стандартный язык запросов: TS=("e-governance" OR "electronic governance" OR 
"egovernance" OR "e-government" OR "electronic government" OR "egovernment" OR "e-participation" OR "electronic participation" OR "eparticipation" OR "e-democracy" OR "electronic democracy" OR "e-democracy"). Период публикации был ограничен 2000 2018 гг., но большинство работы были опубликованы в 2007-2017 гг., при этом примечательно, что количество журнальных статей и трудов конференций оказалось практически одинаковым (табл. 1). Всего по запросу получено 10,5 тыс. документов.

Таблица 1. Количество и структура публикаций по теме в базе данных Web of Science

\begin{tabular}{|c|c|c|c|c|c|}
\hline \multirow{2}{*}{ Год } & \multirow{2}{*}{ Всего } & \multicolumn{2}{|c|}{ Статьи в журналах } & \multicolumn{2}{|c|}{ Российские публикации } \\
\cline { 3 - 6 } & публикаций & количество & процент & количество & процент \\
\hline 2018 & 522 & 382 & 73,2 & 11 & 2,1 \\
2017 & 737 & 417 & 56,6 & 22 & 3,0 \\
2016 & 874 & 403 & 46,1 & 39 & 4,5 \\
2015 & 793 & 398 & 50,2 & 7 & 0,9 \\
2014 & 806 & 428 & 53,1 & 8 & 1,0 \\
2013 & 825 & 465 & 56,4 & 7 & 0,9 \\
2012 & 754 & 453 & 60,1 & 4 & 0,5 \\
2011 & 704 & 375 & 53,3 & 5 & 0,7 \\
2010 & 793 & 354 & 44,6 & 4 & 0,5 \\
2009 & 771 & 274 & 35,5 & 4 & 0,5 \\
2008 & 786 & 290 & 36,9 & 1 & 0,1 \\
2007 & 674 & 274 & 40,7 & 2 & 0,3 \\
2006 & 402 & 194 & 48,3 & 3 & 0,7 \\
2005 & 397 & 217 & 54,7 & 3 & 0,8 \\
2004 & 295 & 152 & 51,5 & 1 & 0,3 \\
2003 & 243 & 161 & 66,3 & 1 & 0,4 \\
2002 & 118 & 85 & 72,0 & 0 & 0 \\
2001 & 57 & 22 & 38,6 & 0 & 0 \\
2000 & 32 & 14 & 43,8 & 0 & 1,2 \\
\hline ВСЕГО & 10583 & 5358 & 50,6 & 122 & \\
\hline
\end{tabular}

Мы видим, что процесс вхождения российских исследователей в международное пространство публикаций имеет позитивную тенденцию: первые публикации в базе Web of Science зафиксированы с 2003 года (2003 и 2004 - по одной публикации в год), а с 2009 ежегодно публикуется не менее 4 статей $(0,5 \%)$ и лишь с 2016 года наметился достаточно значимый рост доли российских статей до 2 - $4 \%$ от массива публикаций за год

Для изучения российской исследовательской повестки мы выбрали единственный институциональный информационный ресурс в России: Научную электронную библиотеку - eLibrary (https://elibrary.ru/). Сбор данных из этой базы ограничен тем, что в системе нет встроенного автоматического инструмента для получения библиографических данных. На основании того же поискового запроса мы сначала сгенерировали выборку из 23 тыс. записей, каждая из которых имеет уникальный идентификатор в электронной библиотеке.

Пришлось предпринять дополнительные действия, чтобы извлечь идентификаторы из подборок, которые были сформированы базой в результате отработки поисковых запросов. Это было сделано с помощью специально сконструированного программного модуля. В результате получился список URL отобранных документов, который был проанализирован на наличие дублетных записей и после этого использовался для скачивания необходимой информации. Скачивание данных осуществлялось с помощью парсинга, в результате чего был получен исходный код HTML-страницы для последующего извлечения нужных полей (тегов) документа. На следующем этапе технологической обработки данных была произведена конвертация извлеченных данных в обменный текстовый формат, используемый Web of Science. В результате весьма трудоемкой технологии был получен 
массив данных, совместимый по основным полям, с выгрузкой из базы Web of Science и пригодный для загрузки в систему VOSviewer.

Сразу следует отметить ограничения и недостатки технологии извлечения данных, к которой мы вынуждены были прибегнуть. Во-первых, это невозможность получения и привязки к документам библиографии, используемой в научном тексте. Во-вторых, неполнота данных по отдельным полям библиографического описания (в некоторых полученных документах, к примеру, отсутствует год издания и др. сведения). В-третьих, отсутствует возможность анализа массива публикаций по типам документов (статьи в журналах, материалы конференций, монографии и т.п.).

Это существенно ограничивает возможности сопоставления результатов анализа двух массивов данных (Web of Science и eLibrary.ru). Другое ограничение заключается в различной информационной политике и отличных критериях отбора у этих двух коллекциях научных публикаций. Критерии отбора в базу РИНЦ (eLibrary.ru) существенно ниже, чем в базу Web of Science, что объясняет разницу в количестве публикаций по теме в условиях, когда в России существенно меньше научных журналов, обращающихся к данной тематике, меньше исследователей и исследовательских центров, меньше регулярных научных конференций. При этом за аналогичный период в российской базе по ключевым словам было отобрано более чем в два раза больше описаний публикаций (23 тыс. против 10,5 тыс. документов из $\mathrm{WoS})$.

С использованием инструментария VOSviewer был проведен анализ ключевых слов (cooccurrence) для международной и российской базы данных, в результате чего была осуществлена оценка интенсивности использования одного термина с другим. Был составлен специальный тезаурус (442 термина) для перевода русских терминов на английский, чтобы объединить похожие термины и устранить опечатки в ключевых словах.

Для построения наукометрической карты мы взяли только те ключевые слова, которые встречаются в выборке не менее 15 раз, сознательно исключая термины запроса, поскольку они присутствуют почти во всех документах и искажают кластеризацию. Таким образом, окончательный выбор ключевых слов состоит из 364 терминов для международной базы данных и 283 терминов для российской базы данных. Минимальный порог для формирования кластера был установлен в 15 терминов для англоязычной и 10 - для российской базы данных.

Кроме того, анализ данных Web of Science позволил произвести кластеризацию публикаций по степени сходства их библиографии (bibliographic coupling), в разрезе институциональной аффилиации авторов. Иными словами, чем больше общих цитат у двух статей на какую-либо третью работу, тем сильнее их связь. В кластеризации отображены организации, которые имеют не менее 5 публикаций и 5 цитат, всего отобрано 582 организации. Минимальный порог кластера установлен в 10 организаций.

\section{4. Результаты обработки и полученные данные}

Визуализация результатов представлена на рис. 1, где размер объекта отражает его общую силу связей (total link strength), а ширина линий - силу связи (link strength) между двумя терминами.

Анализ позволяет выделить несколько кластеров, которые можно условно обозначить как «Инфраструктура» (зеленый), «Принятие новых технологий» (желтый), «Управление» (синий), «Участие» (красный) и «Развитие» (фиолетовый). Интерпретация кластеров основана на встречающихся в них ключевых словах, однако, следует отметить, что данное разделение довольно условно, поскольку и кластеры, и термины взаимосвязаны, зачастую могут пересекаться.

Особенностью кластера «Инфраструктура» является его «технический» уклон в привязке к проблемам государственного администрирования (public administration). B рамках него, в частности, освещаются темы построения архитектуры электронного 
правительства, оптимизации административных процессов, обеспечения безопасности и удобства использования технологий, особенно в контексте электронных услуг. Второй кластер - «Принятие технологий» (acceptance, adoption) объединяет в себе довольно обширную группу работ, посвященных факторам принятия и использования новых технологий. Такие работы, как правило, носят эмпирический, каузальный и количественный характер и используют несколько доминирующих теоретических моделей - Technology Acceptance Model (TAM), IS Success Model, UTAUT Model и др. Особую роль в рамках здесь приобретает концепт доверия, который связан и с другими тематическими направлениями.

An vosviewer

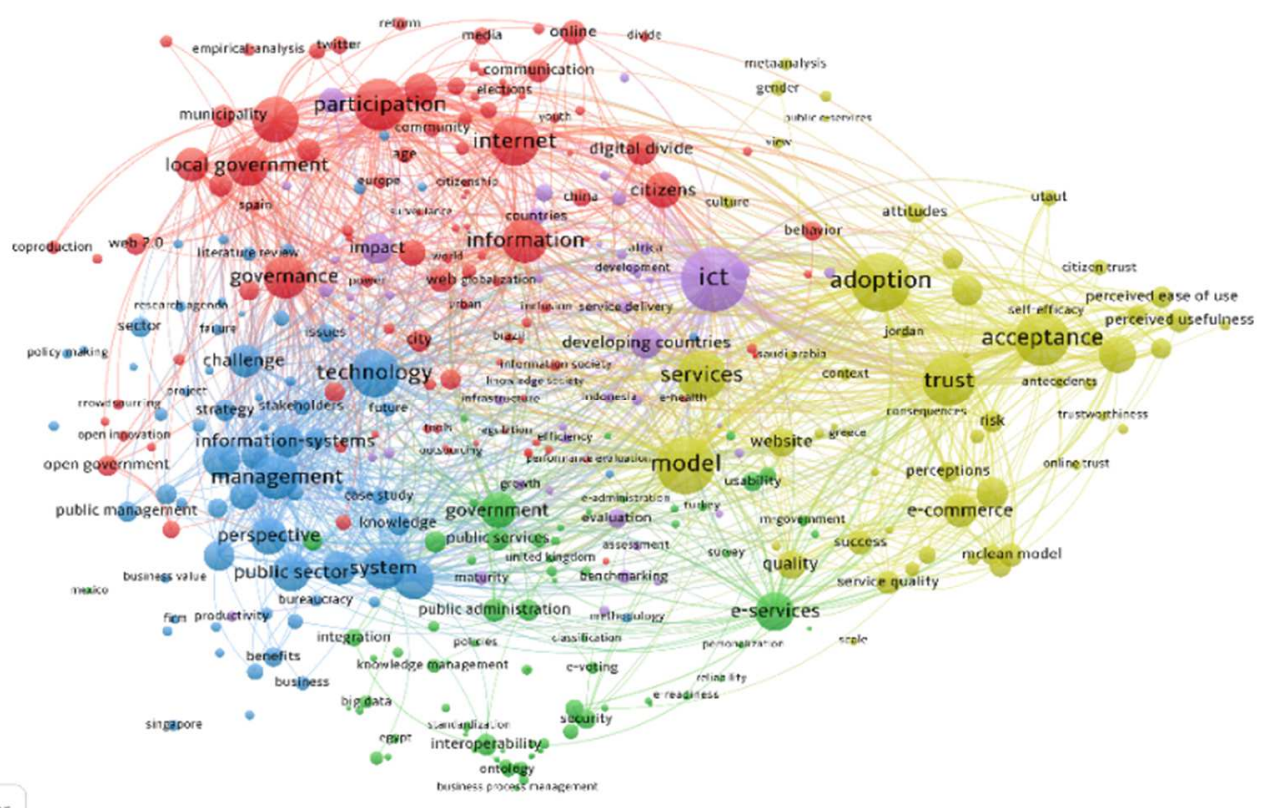

Рис. 1. Библиометрическая карта публикаций в предметной области «Электронное государство» на английском языке (источник: Web of Science; инструментарий: VOSviewer; метод: Keyword Co-occurence)

Кластер «Управление» объединяет группу концептов, связанных с проблемой управления инновациями и цифровыми трансформациями в государственном секторе. Среди прочего, это включает вопросы координации усилий различных государственных и негосударственных акторов, а также стратегического менеджмента. Кластер «Участие», в целом, составляет группу исследований по электронному участию и электронной демократии, представляя довольную широкую группу концептов. Он оказывается ближе к «Управлению» в проблематике открытого правительства и открытых данных, и тяготеет к «Развитию» и «Принятию технологий» в вопросах инклюзивности и равенства. Ключевыми блоками кластера являются вопросы политики и управления, гражданского участия и контроля, местного самоуправления, «умных городов» и социальных медиа.

Своеобразную промежуточную, и в чем-то интегративную роль играет кластер «Развитие», которых охватывает проблематику адаптации и использования новых технологий, их влияния на развивающиеся страны, устойчивое развитие и «хорошее управление».

В целом, область исследований «электронного государства» действительно является мультидисциплинарной областью, в которой есть место как точным наукам, так и 
социально-гуманитарному знанию [10], хотя представляется, что баланс несколько смещен в сторону последнего. Между разными дисциплинами, как показывает кластеризация, наблюдается определенное «разделение труда» в теоретическом и прикладном изучении феномена - собственно от разработки и оптимизации технологий до исследования их влияния на государство и общество. В то же время, о полной интеграции разных дисциплин говорить преждевременно: как показывает анализ, кластеры в основном связаны общими терминами («правительство», «управление», «ИКТ») и гораздо реже - по более узким концептам, где коллаборация разных наук была бы наиболее полезна.

Для анализа институциональной картины изучения «электронного государства» была также построена наукометрическая карта, демонстрирующая силу связи публикаций на основе их библиографических списков (bibliographic coupling), в привязке с аффилиацией авторов. Результаты кластеризации университетов представлены на рис. 2.

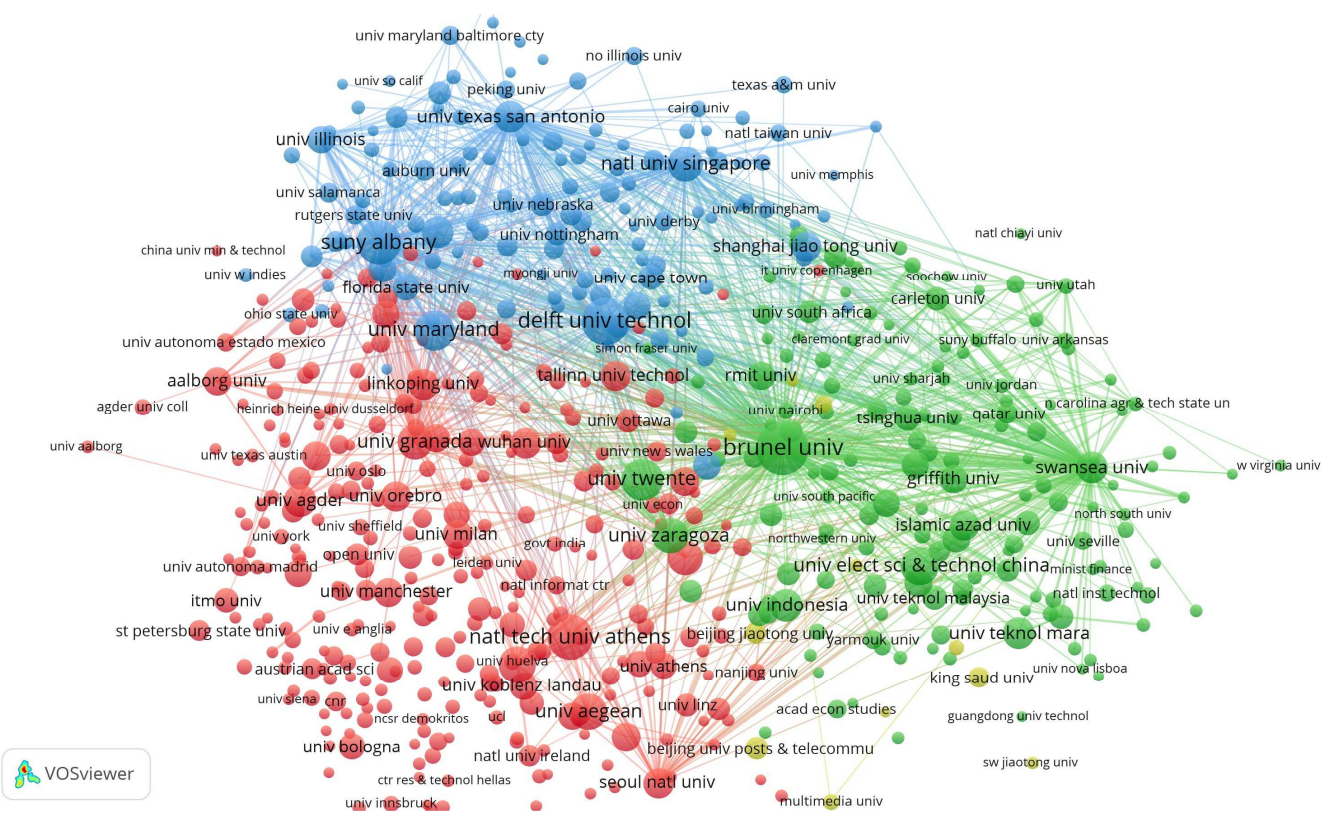

Рис. 2. Библиометрическая карта публикаций в предметной области «Электронное государство» на английском языке (источник: Web of Science; инструментарий: VOSviewer; метод:

Bibiliographic Coupling - Organization)

На карте выделяются несколько университетов, имеющих максимальный «вес»: в синем кластере Университет Штата Нью-Йорк в Олбани (США), Делфтский технический университет (Нидерланды), Университет Мэриленда (США) и Национальный университет Сингапура. Зеленый кластер сформирован вокруг явного лидера - Университета Брунеля (Лондон), красный кластер тоже имеет точку притяжения - Афинский политехнический университет (Греция). Все эти университеты являются лидерами в исследованиях по различным темам, связанным с «электронным государством». Синий кластер и лидирующие в нем университеты известны работами в области электронного правительства (E-Government) и управления (E-Governance), кластер с центром в Университете Брунеля (зеленый) специализируется на работах в области институциональных и административных вопросов и развития связанных с ними технологий электронного управления. Красный кластер более ориентирован на изучение специфики взаимодействия граждан с правительством в рамках электронного участия (E-Participation) и демократии (EDemocracy). Следует отметить, что в исследованном массиве проявились только два 
российских университета - СПбГУ и Университет ИТМО (находятся в красном кластере, хотя у Университета ИТМО прослеживается связь с Университетом Олбани). Конечно, невозможно однозначно определить границы между этими исследовательскими направлениями, а термин «специализация» в данном контексте не очень удачный, однако содержательный анализ публикаций исследователей из обозначенных на карте университетов позволяет сделать такие выводы. Кроме того, помимо тематической дифференциации, проявляется и географическая, где университеты США тяготеют к синему кластеру, европейские - к красному, а зеленый представлен университетами Азии, Ближнего Востока и Африки. Хотя и здесь границы не являются четкими, можно говорить о некоторой регионализации исследований «электронного государства».

Исследование массива библиографии, полученной из базы данных Web of Science, позволило выявить количественные позиции, связанные с вхождением российских исследователей тематики «Электронное государство» в международное научное пространство. Распределение количества и доли публикаций, имеющих аффилиацию с российскими организациями, представлено в табл. 1. Мы видим, что в базе Web of Science первые публикации зарегистрированы начиная с 2003 года и лишь с 2016 года их количество стало хоть как-то значимым (превысило 1\%). Также следует учитывать пилотный характер представляемого исследования и ограниченность базы данных. Хотя при расширении базы, путем включения изданий, индексируемых в Scopus, трудно предположить, что доля библиографических документов российских авторов существенно возрастет даже при увеличении абсолютных цифр.

Подводя промежуточный итог, можно зафиксировать тенденцию роста доли российских публикаций по теме нашего исследования, в мировом потоке библиографии, что видно из анализа данных из Web of Science.

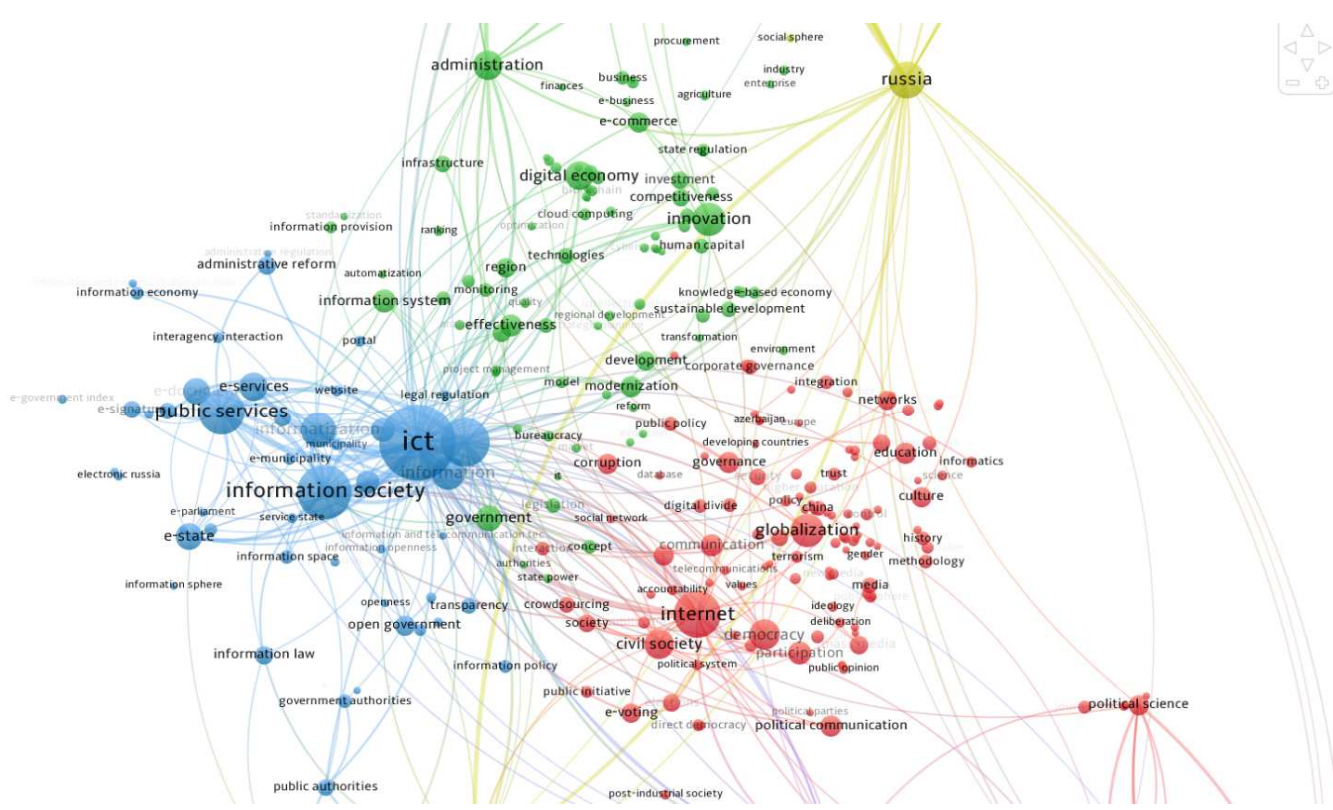

Рис. 3. Библиометрическая карта публикаций в предметной области «Электронное государство» на русском языке (источник: РИНЦ - eLibrary.ru; инструментарий: VOSviewer; метод: Keyword Co-occurence)

Визуализация российского исследовательского поля представлена на рис. 3. На библиометрической карте выделяются три значимых кластера, которые можно обозначить как «Интернет и политика» (красный), «ИКТ в управлении и экономике» (зеленый), и 
«Электронные услуги» (синий). Первый (красный) кластер охватывает проблематику электронного участия и демократии (хотя термин «электронная демократия» употребляется значительно чаще), а также политической коммуникации. Кластер «ИКТ в управлении и экономике» охватывает довольно широкий спектр концептов, связанных с менеджментом и развитием инфраструктуры электронного правительства, а также новую тематику «цифровой экономики». Синий кластер, хотя и содержит такие темы, как «открытое правительство» и «прозрачность», прежде всего сконцентрирован вокруг проблематики электронных услуг и документооборота.

В целом, полученные результаты подтверждают тот факт, что осмысление «электронного государства» в российской науке по большей части происходит с позиций права и социальных наук [13], а дисциплинарный баланс, в отличие от международной повестки дня, существенно смещен в сторону социально-гуманитарного знания. Кроме того, несмотря на больший охват публикаций, их концептуальное содержание не вполне соответствует международной исследовательской программе, некоторые аспекты (например, принятие технологий) в рамках принятых моделей фактически выпадают из поля зрения исследователей. Во всяком случае, такой вывод можно сделать на основании нашей выборки публикаций.

Гораздо более явственно прослеживается взаимосвязь между российскими исследованиями и разными этапами государственной политики построения электронного правительства: электронными услугами (синий кластер), электронным участием (красный кластер) и «цифровой экономикой» (зеленый кластер). Помимо низкой интернационализации российских исследований, описанной выше, причиной подобной ситуации видимо является отсутствие стимулов к производству инновационного и прикладного научного знания. Прежде всего, не хватает спроса со стороны бизнессообщества, где по-прежнему, как и в государственном секторе, часто применяются методы нового государственного менеджмента [22], а также со стороны граждан, поскольку инициативы в области электронного участия и открытого правительства отличаются низкой результативностью [23]. Как результат, единственный стимул исходит от государства, которое искусственно повышает интерес к новым темам, вроде «цифровой экономики», «больших данных» или «умных городов».

\section{5. Заключение}

В данной работе представлены результаты первого этапа библиометрического анализа предметной области, условно обозначаемой «электронное государство». В результате можно сформулировать следующие выводы и рекомендации для продолжения работы на следующих этапах.

1. Библиометрические карты, полученные в результате анализа двух баз данных (международной, полученной из Web of Science, и российской - из базы РИНЦ - eLibrary.ru), позволили вычленить кластеры и связи между ними, характерные для данной предметной области и смежных направлений. Российское пространство публикаций, по сравнению с международным, характеризуется существенной разобщенностью и меньшей связанностью, по сравнению с международным.

2. Интеграция двух исследовательских пространств - международного и российского находится на весьма низком уровне. Обращение к первоисточникам показало, что существенный вклад в усиление данной тенденции вносит активизация участия в международных конференциях европейских и американских университетов, а также организация российских международных научных конференций, с публикацией трудов в изданиях, индексируемых в базах Scopus и Web of Science. Более точные данные смогут быть получены на следующих этапах данного исследования, после расширения информационной базы путем подключения информации о тех публикациях, которые индексируются в базе Scopus, но отсутствуют в Web of Science. В предметном поле 
представляемого исследования имеется существенный массив просидингсов научных конференций, труды которых публикуются в издательстве АСМ (Нью Йорк, США), индексируются в Scopus, однако отсутствуют в базе Web of Science. Это три международные научные конференции - Annual International Conference on Digital Government Research (Dg.o, проводится с 2000 г.); International Conference on Theory and Practice of Electronic Governance (ICEGOV, с 2007 г.) и Electronic Governance and Open Society: Challenges for Eurasia (EGOSE, с 2014 по 2017 в издательстве ACM, с 2018 г. - Springer).

3. Исследование российского публикационного пространства было проведено с существенными ограничениями, которые связаны с отсутствием возможности выгрузить базу с тем набором полей, который используется в базе Web of Science. Это ограничение не позволило провести анализ взаимного цитирования авторов в корпусе публикаций. А тот факт, что база для анализа была сформирована в результате краулинга, создало ряд проблем, что повлияло на полноту извлеченных данных и потребовало исключить ряд документов из массива.

4. При сравнительном исследовании важным аспектом являются параметры сопоставления информации и возможности оперирования данными. На пилотном этапе не ставилась задача создания единой базы и анализ осуществлялся по двум массивам библиографической информации, полученным из двух источников, имеющих различную информационную политику и принципы отбора публикаций. Широта охвата, которая свойственна РИНЦ (eLibrary.ru), обусловила тот факт, что массив российской библиографии оказался в три раза больше, чем полученный из международной базы Web of Science. Для обеспечения лучшей сопоставимости российской базы можно предложить при отборе документов использовать ограничение статьями из журналов, включенных в т.н. «ядро РИНЦ». Однако это ограничение исключит из базы не только многочисленные тезисы докладов, но и монографии и статьи из сериальных изданий и сборников. При этом следует отметить, что для первого этапа библиометрического исследования расширение базы вполне оправдано, т.к. оно позволило обобщенно взглянуть на предметную область, осуществить кластеризацию тематических блоков и увидеть специфику поля российских публикаций в сравнении с международными.

5. Обобщая результаты пилотного исследования, можно предложить один из возможных подходов к организации информационной базы для библиометрии в данной предметной области. Этот подход опирается на формирование информационной базы методами экспертного отбора. Примером такой базы можно считать информационный ресурс DGRL (http://faculty.washington.edu/jscholl/dgrl/), формируемый в Университете Вашингтона известным исследователем электронного правительства Шулем. Этот корпус публикаций можно взять за основу и дополнить массивом библиографии российских авторов (публикации на русском языке). Естественно при этом открытым остается вопрос о том, какими принципами необходимо руководствоваться при отборе публикаций в эту базу. Конечно, желательно соблюсти правила и критерии отбора, аналогичные тем, которые используются при формировании и развитии DGRL. Такой информационный проект можно развивать как партнерский с предоставлением доступа российским исследователям и преподавателям. Важным дополнительным эффектом проекта будет донесение до российских исследователей заявленной тематики стандартов качества научных публикаций, принятых в международном исследовательском сообществе.

Работа выполнена при поддержке РНФ, проект №18-18-00360 «Электронное участие как фактор динамики политического процесса и процесса принятия государственных решений». 


\section{Литература}

[1] Grönlund A., Horan T.A. Introducing e-Gov: History, Definitions, and Issues // Communications of the Association for Information Systems. 2004. Vol. 15 (39). P. 713-729. DOI: $10.17705 / 1 C A I S .01539$.

[2] eGovernment Benchmark 2018 Securing eGovernment for all. European Commission DG Communications Networks, Content \& Technology, 2018. - 200 p. - URL: https://ec.europa.eu/information_society/newsroom/image/document/2018-

47/egovernment_benchmark_2018_background_report_F21FA84B-0254-F4DB7B2FC4567D4AA925_55487.pdf.

[3] Charalabidis Y., Koussouris S. (eds) Empowering Open and Collaborative Governance. Technologies and Methods for Online Citizen Engagement in Public Policy Making. Springer, Berlin, Heidelberg, 2012. DOI: 10.1007/978-3-642-27219-6.

[4] Мелюхин И.С. Информационное общество: истоки, проблемы, тенденции развития. М.: Изд-во МГУ, 1999.

[5] Ракитов А.И. Информация, наука, технология в глобальных исторических изменениях. M., 1998.

[6] Коротков А.В., Кристальный Б.В., Курносов И.Н. Государственная политика Российской Федерации в области развития информационного общества. - М.: ООО «Трейн», 2007.

[7] Reece B. E-government literature review // Journal of E-government. 2006. Vol. 3(1). P. 69110. DOI: $10.1300 / J 399 v 03 n 0105$.

[8] Sæbø Ø., Rose J., Flak L.S. The shape of eParticipation: Characterizing an emerging research area // Government Information Quarterly. 2008. Vol. 25 (3). P. 400-428. DOI: 10.1016/j.giq.2007.04.007.

[9] Ridley G. EGovernment: Making Sense of Fragmentation and Contradiction // E-gov pre-ECIS Workshop, 8 June 2008, Galway Ireland. - URL: https://eprints.utas.edu.au/7062/.

[10]Scholl H.J.J. Discipline or Interdisciplinary Study Domain? Challenges and Promises in Electronic Government Research // Digital Government: E-Government Research, Case Studies, and Implementation. Integrated Series Information Systems. Vol. 17. Springer, Boston, MA, 2008. P. 21-41. doi: 10.1007/978-0-387-71611-4 2.

[11]Coelho T.R., Przeybilovicz E., Cunha M.A., Echternacht T.H.S. Positioning Brazil in International eGov research: a proposal based from literature review // 49th Hawaii International Conference on System Sciences (HICSS). IEEEБ 2016. P. 2677-2686. DOI: 10.1109/HICSS.2016.336.

[12]Dias G.P. A decade of Portuguese research in e-government: evolution, current standing, and ways forward // Electronic Government an International Journal. 2006. Vol. 12(3). P. 201-222. DOI: 10.1504/EG.2016.078415.

[13]Ерженин Р.В. Электронное правительство России: обзор научных публикаций и исследований // Вопросы государственного и муниципального управления. 2018. № 3. C. $205-228$.

[14]Alcaide-Muñoz L., Rodríguez-Bolívar M.P., Cobo M.J., Herrera-Viedma E. Analysing the scientific evolution of e-Government using a science mapping approach // Government Information Quarterly. 2017. Vol. 34 (3). P. 545-555. DOI: 10.1016/j.giq.2017.05.002.

[15]Rodríguez-Bolívar M.P., Alcaide-Muñoz L., Cobo M.J. Analyzing the scientific evolution and impact of e-Participation research in JCR journals using science mapping // International Journal of Information Management. 2018. Vol. 40. P. 111-119. DOI: 10.1016/j.ijinfomgt.2017.12.011.

[16]Jafarjalali S.M. Visualizing e-government emerging and fading themes using SNA techniques // 10th International Conference on e-Commerce in Developing Countries: with focus on eTourism (ECDC). IEEE, 2016. DOI: 10.1109/ECDC.2016.7492983. 
[17]Khan G.F., Park H.W. The e-government research domain: A triple helix network analysis of collaboration at the regional, country, and institutional levels // Government Information Quarterly. 2013. Vol. 30 (2). P. 182-193. DOI: 10.1016/j.giq.2012.09.003.

[18]Qi T., Wang T., Ma Y., Zhang W., Zhu Y. A scientometric analysis of e-participation research // International Journal of Crowd Science. 2018. Vol. 2 (2). P. 136-148. DOI: 10.1108/IJCS08-2018-0015.

[19]Scholl H.J.J., Dwivedi Y.K. Forums for electronic government scholars: Insights from a 2012/2013 study // Government Information Quarterly. 2014. Vol. 31 (2). P. 229-242. DOI: 10.1016/j.giq.2013.10.008.

[20]Van Eck N.J., Waltman L. Software survey: VOSviewer, a computer program for bibliometric mapping // Scientometrics. 2010. Vol. 84 (2). P. 523-538. DOI: 10.1007/s11192-009-0146-3.

[21]Van Eck N.J., Waltman L., Dekker R., van den Berg J. A comparison of two techniques for bibliometric mapping: Multidimensional scaling and VOS // Journal of the American Society for Inf. Sc. and Tech. 2010. Vol. 61 (12). P. 2405-2416. DOI: 10.1002/asi.21421.

[22]Chugunov A.V., Kabanov Y., Zenchenkova K.: Russian e-Petitions Portal: Exploring Regional Variance in Use // Tambouris E. et al. (eds) Electronic Participation. ePart 2016. LNCS. Vol. 9821. Springer, Cham, 2016. P. 109-122. DOI: 10.1007/978-3-319-45074-2_9.

[23]Клименко А.В. Десятилетие административной реформы: результаты и новые вызовы // Вопросы государственного и муниципального управления. 2014. № 1. С. 8-51.

\title{
"Electronic Governance" As an Interdisciplinary Scientific Field: Scientometrics Analysis
}

\author{
Andrei V. Chugunov, ${ }^{1}$ Yury Kabanov ${ }^{1,2}$ \\ ${ }^{1}$ ITMO University \\ ${ }^{2}$ National Research University Higher School of Economics
}

The object of the research is an interdisciplinary research field of e-governance. Here we deal with the two major tasks - to assess the conceptual coherence of the field, as well as to explore the interrelationship between the major research context, especially in relation to the new emerging research issues, like e-participation. To solve these tasks, we use scientometrics approach and the automated analysis of publications in English (the Web of Science database) and Russian (RSCI eLibrary.ru). To cluster and visualize the data we use the VOSviewer software, which allows to analyze the large amount of bibliometric data and perform science mapping. The results obtained demonstrate that the global agenda on e-governance is a balanced research area, comprising of hard and soft science issues, though the integration between various research contexts is required. At the same time, the Russian agenda is lagging behind due to low internationalization and lack of internal stimuli for science production.

Keywords: e-governance, scientometrics, bibliometrics, scientific publications, Web of Science, RSCI

Reference for citation: Chugunov A.V., Kabanov Y. "Electronic Governance" As an Interdisciplinary Scientific Field: Scientometrics Analysis // The State and Citizens in the Electronic Environment. Vol. 3 (Proceedings of the XXII International Joint Scientific Conference «Internet and Modern Society», IMS-2019, St. Petersburg, June 19-22, 2019). - St. Petersburg: ITMO University, 2019. P. 11 - 24. DOI: 10.17586/2541-979X-2019-3-11-24 


\section{Reference}

[1] Grönlund A., Horan T.A. Introducing e-Gov: History, Definitions, and Issues // Communications of the Association for Information Systems. 2004. Vol. 15 (39). P. 713-729. DOI: $10.17705 / 1 C A I S .01539$.

[2] eGovernment Benchmark 2018 Securing eGovernment for all. European Commission DG Communications Networks, Content \& Technology, 2018. - 200 p. - URL: https://ec.europa.eu/information_society/newsroom/image/document/2018-

47/egovernment_benchmark_2018_background_report_F21FA84B-0254-F4DB7B2FC4567D4AA925_55487.pdf.

[3] Charalabidis Y., Koussouris S. (eds) Empowering Open and Collaborative Governance. Technologies and Methods for Online Citizen Engagement in Public Policy Making. Springer, Berlin, Heidelberg, 2012. DOI: 10.1007/978-3-642-27219-6.

[4] Meljuhin I.S. Informacionnoe obshhestvo: istoki, problemy, tendencii razvitija. M.: Izd-vo MGU, 1999.

[5] Rakitov A.I. Informacija, nauka, tehnologija v global'nyh istoricheskih izmenenijah. M., 1998.

[6] Korotkov A.V., Kristal'nyj B.V., Kurnosov I.N. Gosudarstvennaja politika Rossijskoj Federacii v oblasti razvitija informacionnogo obshhestva. — M.: OOO «Trejn», 2007.

[7] Reece B. E-government literature review // Journal of E-government. 2006. Vol. 3(1). P. 69110. DOI: 10.1300/J399v03n01_05.

[8] Sæbø Ø., Rose J., Flak L.S. The shape of eParticipation: Characterizing an emerging research area // Government Information Quarterly. 2008. Vol. 25 (3). P. 400-428. DOI: 10.1016/j.giq.2007.04.007.

[9] Ridley G. EGovernment: Making Sense of Fragmentation and Contradiction // E-gov pre-ECIS Workshop, 8 June 2008, Galway Ireland. - URL: https://eprints.utas.edu.au/7062/.

[10]Scholl H.J.J. Discipline or Interdisciplinary Study Domain? Challenges and Promises in Electronic Government Research // Digital Government: E-Government Research, Case Studies, and Implementation. Integrated Series Information Systems. Vol. 17. Springer, Boston, MA, 2008. P. 21-41. doi: 10.1007/978-0-387-71611-4_2.

[11]Coelho T.R., Przeybilovicz E., Cunha M.A., Echternacht T.H.S. Positioning Brazil in International eGov research: a proposal based from literature review // 49th Hawaii International Conference on System Sciences (HICSS). IEEEБ 2016. P. 2677-2686. DOI: 10.1109/HICSS.2016.336.

[12]Dias G.P. A decade of Portuguese research in e-government: evolution, current standing, and ways forward // Electronic Government an International Journal. 2006. Vol. 12(3). P. 201-222. DOI: 10.1504/EG.2016.078415.

[13]Erzhenin R.V. Jelektronnoe pravitel'stvo Rossii: obzor nauchnyh publikacij i issledovanij // Voprosy gosudarstvennogo i municipal'nogo upravlenija. 2018. № 3. S. 205 - 228.

[14]Alcaide-Muñoz L., Rodríguez-Bolívar M.P., Cobo M.J., Herrera-Viedma E. Analysing the scientific evolution of e-Government using a science mapping approach // Government Information Quarterly. 2017. Vol. 34 (3). P. 545-555. DOI: 10.1016/j.giq.2017.05.002.

[15]Rodríguez-Bolívar M.P., Alcaide-Muñoz L., Cobo M.J. Analyzing the scientific evolution and impact of e-Participation research in JCR journals using science mapping // International Journal of Information Management. 2018. Vol. 40. P. 111-119. DOI: 10.1016/j.ijinfomgt.2017.12.011.

[16]Jafarjalali S.M. Visualizing e-government emerging and fading themes using SNA techniques // 10th International Conference on e-Commerce in Developing Countries: with focus on eTourism (ECDC). IEEE, 2016. DOI: 10.1109/ECDC.2016.7492983.

[17]Khan G.F., Park H.W. The e-government research domain: A triple helix network analysis of collaboration at the regional, country, and institutional levels // Government Information Quarterly. 2013. Vol. 30 (2). P. 182-193. DOI: 10.1016/j.giq.2012.09.003. 
[18]Qi T., Wang T., Ma Y., Zhang W., Zhu Y. A scientometric analysis of e-participation research // International Journal of Crowd Science. 2018. Vol. 2 (2). P. 136-148. DOI: 10.1108/IJCS08-2018-0015.

[19]Scholl H.J.J., Dwivedi Y.K. Forums for electronic government scholars: Insights from a 2012/2013 study // Government Information Quarterly. 2014. Vol. 31 (2). P. 229-242. DOI: 10.1016/j.giq.2013.10.008.

[20]Van Eck N.J., Waltman L. Software survey: VOSviewer, a computer program for bibliometric mapping // Scientometrics. 2010. Vol. 84 (2). P. 523-538. DOI: 10.1007/s11192-009-0146-3

[21]Van Eck N.J., Waltman L., Dekker R., van den Berg J. A comparison of two techniques for bibliometric mapping: Multidimensional scaling and VOS // Journal of the American Society for Inf. Sc. and Tech. 2010. Vol. 61 (12). P. 2405-2416. DOI: 10.1002/asi.21421.

[22]Chugunov A.V., Kabanov Y., Zenchenkova K.: Russian e-Petitions Portal: Exploring Regional Variance in Use // Tambouris E. et al. (eds) Electronic Participation. ePart 2016. LNCS. Vol. 9821. Springer, Cham, 2016. P. 109-122. DOI: 10.1007/978-3-319-45074-2_9.

[23]Klimenko A.V. Desjatiletie administrativnoj reformy: rezul'taty i novye vyzovy // Voprosy gosudarstvennogo i municipal'nogo upravlenija. 2014. № 1. S. 8-51. 